International Journal of Biological Sciences

ISSN 1449-2288 www.biolsci.org 2008 4(4):200-201

Letter to the editor

(C) Ivyspring International Publisher. All rights reserved

\title{
New amyloid plaques or a game of hide-and-seek?
}

\section{Kun Zou' ${ }^{1}$, Tomoji Maeda', Makoto Michikawa², and Hiroto Komano ${ }^{1}$}

1. Department of Neuroscience, Faculty of Pharmaceutical Sciences, Iwate Medical University, Yahaba, Iwate 028-3694, Japan

2. Department of Alzheimer's Disease Research, National Institute for Longevity Sciences, National Center for Geriatrics and Gerontology, 36-3 Gengo, Morioka, Obu, Aichi 474-8522, Japan.

Correspondence to: Kun Zou, TEL: 81-19-698-1820; FAX: 81-19-698-1864; Email: kunzou@iwate-med.ac.jp

Received: 2008.06.17; Accepted: 2008.07.07; Published: 2008.07.09

Melanie Meyer-Luehmann et al. recently reported that amyloid plaques form extremely rapidly-within one day, in three mouse models of Alzheimer's disease studied longitudinally in vivo [1]. However, the 'rapid appearance' of 'newborn' amyloid plaques can also be the result of delayed visualization of existing amyloid plaques, which is caused by the increase in the concentration of methoxy-XO4, an amyloid indicator (Fig. 1). The fact that the authors did not find any growth of the 'newborn' amyloid plaques supports this notion.

Most compounds taken up by brain show different regional and time-dependent distributions in the human brain revealed by PET $[2,3]$ and the time for reaching peak concentrations of compounds in CNS can vary from minutes to days after administration [3]. It is unclear, however, whether methoxy-XO4 is distributed evenly across the brain and when the brain concentration of methoxy-XO4 reaches the peak during the 1-7 day time course after an intraperitoneal injection. Using multiphoton microscopy, the kinetics of another amyloid indicator, Pittsburgh compound B (PIB) was studied in an Alzheimer's disease mouse model using an intravenous injection. The uneven distribution and the concentration change of PIB were clearly shown in the small brain areas, particularly in the vicinity of small blood vessels. Interestingly, the concentration of PIB in some small areas even kept increasing after their neighbor areas reached the concentration peak of PIB, indicating that the perfusion of PIB from the high concentration area to the low concentration area takes place all the time in brain [4]. While uneven distribution of methoxy- $\mathrm{XO} 4$ in the brain may occur after an intraperitoneal injection, this distribution is not comparable to PIB because within minutes PIB levels reach saturation across the brain after an intravenous injection. However, the kinetics of intraperitoneally-administered methoxy-XO4 in the blood and the brain should be studied, in particular, an intraperitoneal injection of a $\sim 5,000$-fold higher dose of amyloid indicator than the doses used in PET or SPECT studies may provide a high concentration pool of methoxy- $\mathrm{XO} 4$ in the abdominal cavity and delay methoxy-XO4 to reach its peak in certain brain areas. From our point of view, it is still early to conclude that newly visualized amyloid plaques are 'newborn' before confirming that the concentration of methoxy-XO4 does not increase in the area where 'newborn' amyloid plaques appear.

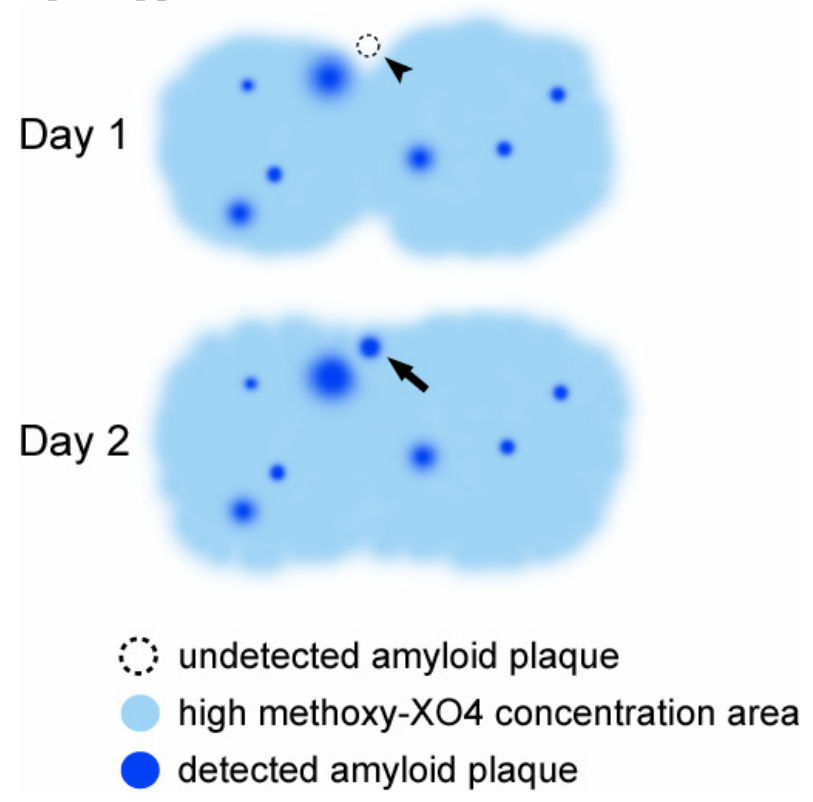

Fig.1. A possible interpretation for 'rapid appearance' of amyloid plaques. An existing amyloid plaque not detected in day 1 can be detected by the increase in the concentration of methoxy-XO4 in day 2. Arrowhead, undetected amyloid plaque; Arrow, detected amyloid plaque. 


\section{Conflict of Interest}

The authors have declared that no conflict of interest exists.

\section{References}

1. Meyer-Luehmann M, Spires-Jones TL, Prada C, et al. Rapid appearance and local toxicity of amyloid-beta plaques in a mouse model of Alzheimer's disease. Nature 2008; 451: 720-4.

2. Small GW, Bookheimer SY, Thompson PM, et al. Current and future uses of neuroimaging for cognitively impaired patients. Lancet Neurol. 2008; 7: 161-72.

3. Muldoon LL, Soussain C, Jahnke K, et al. Chemotherapy delivery issues in central nervous system malignancy: a reality check. J Clin Oncol. 2007; 25: 2295-305.

4. Bacskai BJ, Hickey GA, Skoch J, et al. Four-dimensional multiphoton imaging of brain entry, amyloid binding, and clearance of an amyloid-beta ligand in transgenic mice. Proc Natl Acad Sci U S A. 2003; 100: 12462-67. 